\begin{tabular}{|l|l|l|l|}
\hline Eiszeitalter u. Gegenwart & $\mathbf{3 2}$ & $\begin{array}{c}203-212 \\
1 \mathrm{Abb} ., 2 \mathrm{Tab} .\end{array}$ & Hannover 1982 \\
\hline
\end{tabular}

\title{
Paläoböden der Paderborner und Briloner Hochfläche
}

\author{
ECKE von ZEZSCHWITZ *)
}

Pedogenesis, paleosol, terra calcis, terra fusca, terra rossa, Upper Pleistocene, weathering, marly limestone, Cenoman, Turon, Lower Gretaceous, soil erosion, Pliocene, Pleistocene, plateau, size distribution, major element analysis. Rhine Westphalian Bassin, Tableland Paderborn, Tableland Brilon, Nordrhein-Westfalen, TK 25 Nr.: 4419, 4617

K u rzfassung: Auf den Plateaus der Paderborner Hochfläche haben sich im Jungpleistozän Terrae calcis entwickelt. Aus den Mergelkalksteinen des Cenoman 2 sind Hottensteinverwitterungsdecken hervorgegangen. Die Mergelkalksteine des Turon 2 und 3 sind demgegenüber zu Böden vom Typ der Terra fusca verwittert. Beide Bodenbildungen treten nur fossil auf; sie sind von Lößlehmen bedeckt.

Bei den Terrae calcis der Briloner Hochfläche handelt es sich dagegen meist um praecenomane Bildungen. Hier sind Böden vom Typ der Terra rossa und Terra fusca in Taschen und Spalten des Massenkalks durch cenomane Ablagerungen plombiert worden. Erst im Pliozän und Pleistozän sind diese Bodenbildungen infolge von Abtragungsvorgängen exhumiert worden.

\section{[Paleosols of the Paderborn and Brilon Tablelands]}

Abstract: On the plateaus of the tableland of Paderborn "terrae calcis" developed during the period of younger pleistocene. On the marly-limestones of the "Cenoman 2", called "Hottensteinverwitterungsdecken", were produced. In opposite of this on the marly-limestones of the "Turon 2 and 3 " "terra fusca" developed. These formations of soil can be found only in fossil positions; they are covered by loess-loam.

On the contrary the „terrae calcis“ of the tableland of Brilon are most built during a "praecenoman-period". Here, soils of the "terra rossa" and "terra fusca"-type were conserved in pockets and crevasses of the "Massenkalk" (limestone). But during the "pliocene“ and "pleistocene" period these soils were laid by erosion.

\section{Einleitung}

Schon Mückenhausen \& WORTMAnN (1958) stießen bei bodenkundlichen Übersichtskartierungen im Gebiet der aus cenomanen und turonen Gesteinen aufgebauten Paderborner Hochfläche auf Relikte von Paläoböden vom Typ der Terrae calcis. In den darauffolgenden Jahren bot sich dann Gelegenheit, bei den vom Geologischen Landesamt NordrheinWestfalen zum Zwecke der forstlichen Standorterkundung durchgeführten großmaßstäbigen Bodenkartierungen (v. Zezschwitz 1958, 1964, 1970, 1975; PAAs \& v. Zezschwitz 1972) diese und andere auf der Paderborner Hochfläche vorkommenden Paläoböden zu erforschen.

Die Bodenkarten im Maßstab 1:10 000 der Staatlichen Forstämter Dalheim, Böddeken und Wünnenberg sowie des Stiftsforstes Büren und des Gemeindewaldes Nettelstädt, ergänzt durch Feststellungen im Graf v. Westfalen'schen Forstamt Fürstenberg, zeigen Vorkommen, Verbreitung und typische Vergesellschaftung der Paläoböden dieses Gebietes und lassen auf deren mutmaßliche Genese schließen.

Gemessen am Kenntnisstand über die Paläoböden der Paderborner Hochfläche kann von einer Erforschung der Paläoböden der Briloner Hochfläche noch kaum gesprochen

*) Anschrift des Autors: Dr. E. v. Zezschwitz, Geologisches Landesamt NordrheinWestfalen, De-Greiff-Str. 195, 4150 Krefeld. 
werden. Die in diesem Beitrag diskutierten Befunde stützen sich nämlich nicht auf Kartierungen, sondern sind das Ergebnis zufälliger Einzelbeobachtungen. Wenn trotzdem das Gebiet der Briloner Hochfläche bei der Behandlung der Paläoböden ostwestfälischer Hochebenen in das Thema einbezogen wurde, so deshalb, weil beide Hochflächen (im Ortsbereich von Bleiwäsche) zusammenstoßen und die cenomanen Ablagerungen sich noch heute bis weit auf die höher gelegene, aus mitteldevonischem Massenkalk aufgebaute Briloner Hochfläche erstrecken.

Im übrigen ist die Frage nach der Herkunft der dem Massenkalk aufliegenden Verwitterungsdecken schon früh gestellt worden (PAEckelmann $1931 \mathrm{a}$ ), ohne daß es indessen seither möglich war, sie erschöpfend zu beantworten (FINkE 1971). Die Mitteilung von weiteren Einzelergebnissen soll es ermöglichen, Ansatzpunkte zukünftiger Untersuchungen aufzuzeigen.

\subsection{Paläoböden der Paderborner Hochfläche}

\subsubsection{Hottenstein - Schlufflehm decken}

Auf den von Mergelkalksteinen des Cenoman 2 gebildeten Plateaus befinden sich weit verbreitet mächtige fossile Verwitterungsdecken, die sich durch einen sehr hohen Gehalt an Hottensteinen auszeichnen. Diese als Hottenstein-Schlufflehmdecken in die Literatur eingeführten Substrate sind aus Frostschutt, der kryoturbat und/oder solifluktiv mit älteren Lössen vermengt wurde, hervorgegangen (v. Zezschwitz 1967). Aufgrund der Erstreckung der Plänerschotterströme bis hin zur Lippe und deren Verzahnungen mit anderen pleistozänen Bildungen (LOTZE 1951) ist die Entstehung der Frostwanderschuttdecken in die Saale-Kaltzeit zu stellen. Ausgangs der Saale-Kaltzeit mögen auf den Plateaus bis zu 4 m mächtige Reste der mit Löß vermengten Schuttdecken verblieben sein. Im Bereich von flachen Spülmulden, die sich teilweise weit auf die Plateaus hinauf erstrecken, sind die Schuttdecken sogar heute noch bis zu $5 \mathrm{~m}$ mächtig (MNICH 1979). Während des Eems unterlagen sie der Lösungsverwitterung und Verlehmung. Auf diese Weise wurden die Mergelkalksteine des Frostwanderschutts oder die gleichsam in der Lößmatrix schwimmenden Mergelkalksteine unter Erhaltung ihrer typischen Plänergestalt langsam entcarbonatisiert. Aus den Mergelkalksteinen entstanden Hottensteine.

Eingangs der Weichsel-Kaltzeit wurden die Hottenstein-Schlufflehmdecken erneut von Solifluktionsvorgängen erfaßt, wenngleich in bedeutend geringerem Ausmaß als während der Saale-Vereisung. Hochglazial sedimentierte wiederum Löß. Dessen von der Erosion belassene Verlehmungsprodukte bedecken die hottensteinigen Paläoböden im allgemeinen nur noch schleierartig in Mächtigkeiten von 2 bis $5 \mathrm{dm}$. Diese Decklehme sind meist nur schwach hottensteinig, ein Zeichen dafür, daß die fossilen Hottenstein-Schlufflehmdecken von den Solifluktionsphasen am Ende der Weichsel-Kaltzeit kaum mehr betroffen waren, während die Lösse und deren Verwitterungsprodukte als Fließerden verlagert wurden und dabei Hottensteine aus dem Unterboden aufnahmen.

STILle (1903) hat wohl als einer der ersten über das Vorkommen und die massenhafte Verbreitung der Hottensteine auf der südlichen Paderborner Hochfläche (Cenoman 2) berichtet. Seither ist dem Hottenstein wenig Aufmerksamkeit gewidmet worden. Nur bei Arnold (1965) finden sich einige gesteinsphysikalische Daten, die von Hesemann (1975) übernommen wurden.

Nach Scherp (1979) besteht der die typischen Hottensteine liefernde CenomanPläner (Bl 4419 Kleinenberg, R 349153, H 571104) zu 56,2 Gew.\% aus Calcit und zu $22,8 \mathrm{Gew} . \%$ aus Quarz sowie zu etwa je $1 \%$ aus Feldspat, $3 \%$ aus Kaolinit und $17 \%$ aus Illit und Illit-Montmorillonit, wovon etwa $3 \%$ auf Glaukonit entfallen, der röntgen- 
diffraktometrisch als Illit erfaßt wird. Der Illit-Montmorillonit liegt als MixedlayerMineral mit regelmäßiger Wechsellagerung von Illit und Montmorillonit vor. Calcitfreies Material (durch Behandlung mit Monochloressigsäure aus dem Pläner gewonnen) besteht zu 52 Gew.\% aus Quarz; der Rest im wesentlichen aus Tonmineralen. Aufgrund der zahlreichen in der mikritischen Calcitgrundmasse liegenden carbonatischen Fossilien und Fossilbruchstücke ist das Gestein als glaukonithaltiger biomikritischer Mergelstein zu bezeichnen.

In den Hottensteinen (Bl. 4419 Kleinenberg, R 349148, H 571187) beträgt der diffraktometrisch festgestellte Quarzanteil demgegenüber 60\%. Der Tonanteil ist im Vergleich zum künstlich entkalkten Mergelstein bedeutend geringer (höchstens 15\%). Der Illit-Montmorillonit zeigt dabei eine sehr viel stärkere Abnahme als der Kaolinit. Die restlichen Bestandteile des Hottensteins (etwa 25 bis $30 \%$ ) sind röntgenamorph und bestehen, nach den Dünnschliffen zu urteilen, wahrscheinlich überwiegend aus amorpher Kieselsäure. Diese bildet einen Teil der Grundmasse des Hottensteins, in die außer den Tonmineralen zahlreiche angerundete Quarze von 0,03 bis $0,08 \mathrm{~mm} \phi$ eingebettet sind. Das Gestein besitzt sehr viele Mikroporen (nach ARNOLD 1965 bis zu über 50\%), die infolge herausgelöster Fossilien meist rundliche Formen aufweisen.

Hottensteine sind demnach aus dem Cenoman-Pläner durch Auslaugungs- und Verwitterungsprozesse entstanden. Als Folge dieser Vorgänge hat bei der Umwandlung des Pläners zum Hottenstein der Anteil des Illit-Montmorillonit deutlich abgenommen. Auf-grund der Untersuchungsergebnisse liegt es deshalb nahe anzunehmen, daß die Bildung der Hottensteine dadurch verursacht wird, daß nach der Entcarbonatierung die bei der Verwitterung der Tonminerale freiwerdende Kieselsäure in amorpher Form im Gestein verbleibt und eine Art Gerüst bildet, während die Kationen in Lösung weggeführt werden.

Abrieb von Hottensteinen besteht nahezu vollständig aus Schluff (vgl. Tab. 1, Spalte 4). Das zwischen den Hottensteinen der Hottenstein-Schlufflehmdecken befindliche Bodenmaterial besteht dagegen, wie die zahlreichen in Tab. 1 zusammengestellten Ergebnisse von Schlämmanalysen zeigen, bodenartlich aus schluffig-tonigem Lehm. Die Tonsubstanz kann verschiedener Herkunft sein. Teilweise stammt sie sicherlich aus den eingemengten verlehmten saaleeiszeitlichen Lössen. Substanzen in der Größenordnung der Tonfraktion werden daneben aber auch aus dem Gesteinszerreibsel freigeworden sein, das während der Solifluktionsphasen in den Schuttdecken entstand und deren Mobilität bewirkte.

Nach den Untersuchungsergebnissen von SCHERP (1979) zu urteilen, dürfte im wesentlichen Quarz freigeworden sein, Tonminerale dagegen nur untergeordnet. Die an 12 Bodenproben des aus schluffig-tonigem Lehm bestehenden $Z$ wischenmittels der HottensteinSchlufflehmdecken erzielten röntgenographischen Untersuchungsergebnisse*) sprechen für eine derartige Zusammensetzung der Tonfraktion. Außer einem rund 20\%igen röntgenamorphen Rest besteht diese in allen Proben weitgehend übereinstimmend aus etwa $60 \%$ Quarz, 5 bis $10 \%$ Serizit und Illit, 5\% Chlorit, 5\% Montmorillonit und $3 \%$ Feldspat, der vorzugsweise den älteren Lössen entstammen dürfte.

Die in Tab. 2 zusammengestellten T- und S-Werte bestätigen die vermuteten $\mathrm{Zu}$ sammenhänge. Mit rund $13 \mathrm{mval} / 100 \mathrm{~g}$ Boden ist der schluffig-tonige Lehm ungewöhnlich sorptionsschwach, ein Zeichen dafür, daß in der Tonfraktion neben Tonmineralen nicht unerhebliche Quarzanteile vorhanden sein müssen. Unter Abnahme der Basensättigung $(95 \%$ im IICv-Horizont $\rightarrow 61 \%$ im IICvBv-Horizont $\rightarrow$ bis $<10 \%$ im IIfBv-Hori-

*) Für die Durchführung der röntgenographischen Untersuchungen wird Herrn Dr. G. StadLER, Geologisches Landesamt Nordrhein-Westfalen, Krefeld, gedankt. 
Tab. 1: Körnungen von Paläoböden der Paderborner und Briloner Hochfläche

\begin{tabular}{|c|c|c|c|c|c|c|c|c|c|c|c|c|c|}
\hline \multirow{2}{*}{ Gebiet } & \multirow{2}{*}{ Vorkommen } & \multirow{2}{*}{$\begin{array}{c}\text { geologisches } \\
\text { Ausgangs- } \\
\text { material }\end{array}$} & \multicolumn{2}{|c|}{ Bodenbildung } & \multirow[b]{2}{*}{$\underset{0,002}{<}$} & \multicolumn{5}{|c|}{$\begin{array}{l}\text { Korngrößenzusammensetzung } \\
\text { in } \% \text { des Feinbodens }<2 \mathrm{~mm}\end{array}$} & \multirow[b]{2}{*}{$\begin{array}{l}0,6 \\
-2,0 \\
\end{array}$} & \multirow{2}{*}{$\begin{array}{l}\text { Boden- } \\
\text { art }\end{array}$} & \multirow{2}{*}{$\begin{array}{l}\text { Anzahl } \\
\text { unter- } \\
\text { suchte } \\
\text { Proben }\end{array}$} \\
\hline & & & $\begin{array}{c}\text { Substrat } \\
\text { oder Bodentyp }\end{array}$ & Horizont & & $\begin{array}{c}0,002 \\
-0,006\end{array}$ & $\begin{array}{l}0,006 \\
-0,02\end{array}$ & $\begin{array}{c}0,02 \\
-0,06\end{array}$ & $\begin{array}{l}0,06 \\
-0,2\end{array}$ & $\begin{array}{l}0,2 \\
-0,6\end{array}$ & & & \\
\hline \multirow{6}{*}{$\begin{array}{c}\text { Paderborner } \\
\text { Hochfläche }\end{array}$} & \multirow{4}{*}{$\begin{array}{c}\text { weit ver- } \\
\text { breitet auf } \\
\text { Plateaus vor- } \\
\text { kommend }\end{array}$} & \multirow{4}{*}{$\begin{array}{l}\text { Mergelkalk- } \\
\text { steine des } \\
\text { Cenoman } 2\end{array}$} & \multirow{3}{*}{$\begin{array}{c}\text { verbraunte } \\
\text { Hottenstein- } \\
\text { Schlufflehm- } \\
\text { Decke }\end{array}$} & $==\begin{array}{c}==ニ= \\
\\
\operatorname{IIfB}_{\mathrm{v}}\end{array}$ & 35 & 14 & 21 & 26 & 3 & 1 & 一 & utL & 38 \\
\hline & & & & IIC $_{v} B_{v}$ & 38 & 16 & 23 & 20 & 2 & 1 & 一 & utL & 6 \\
\hline & & & & $\mathrm{IIC}_{\mathrm{v}}$ & 40 & 16 & 22 & 16 & 4 & 1 & 1 & utL & 5 \\
\hline & & & Hottensteine & & 3 & 18 & 39 & 40 & - & - & - & $\mathrm{U}$ & 1 \\
\hline & $\begin{array}{l}\text { vereinzelt auf } \\
\text { Plateaus }\end{array}$ & $\begin{array}{c}\text { Kalksteine des } \\
\text { Cenoman } 3\end{array}$ & Terra fusca & $\operatorname{IIfB}_{\mathrm{v}}$ & 63 & 12 & 11 & 13 & 1 & - & - & $1 \mathrm{~T}^{\circ}$ & 4 \\
\hline & $\begin{array}{l}\text { weit ver- } \\
\text { breitet auf } \\
\text { Plateaus }\end{array}$ & \begin{tabular}{|c|} 
Mergelkalk- \\
steine des \\
Turon 2, 3 u. 4 \\
\end{tabular} & Terra fusca & $\operatorname{IIfB}_{v}$ & 56 & 16 & 13 & 14 & 1 & - & - & $1 \mathrm{~T}$ & 37 \\
\hline Briloner & in Steinbrü- & Kalksteine des & Terra fusca & $\operatorname{IIfB}_{v}$ & 66 & 8 & 7 & 10 & 3 & 3 & 3 & $\mathrm{~T}$ & 5 \\
\hline Hochfläche & \begin{tabular}{|} 
bau-Auf- \\
schlüssen und \\
Kabelgräben
\end{tabular} & $\begin{array}{c}\text { devons } \\
\text { (Massenkalk) }\end{array}$ & Terra rossa & $\begin{array}{l}\text { aus } 28 \mathrm{~m} \\
\text { Tiefe }\end{array}$ & 79 & 8 & 4 & 7 & 1 & 1 & - & $\mathrm{T}$ & 1 \\
\hline
\end{tabular}


Tab. 2: Chemische Kennwerte von Paläoböden der Paderborner und Briloner Hochfläche

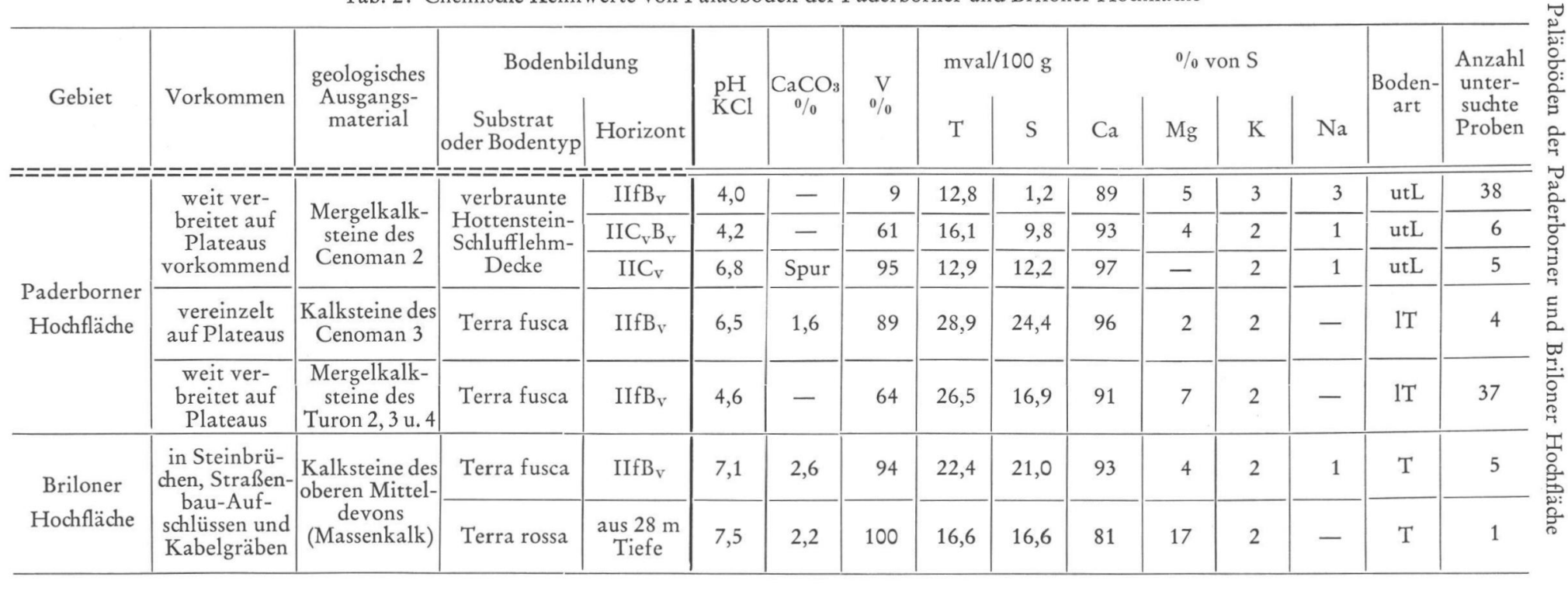


zont) sind die Hottenstein-Schlufflehmdecken verbraunt. Begraben unter jüngeren Lößfließerden oder schleierartig vorhandenen Decklehmen stellen sie das fossile Relikt eines eem- und weichselzeitlich entstandenen Paläobodens dar, der, obwohl aus Oberkreidekalken hervorgegangen, mit $\mathrm{pH}-$ Werten von 4,0 stark sauer ist. Abb. 1 zeigt die kartierten Verbreitungs-Areale dicser Bodenbildung, die im Gebiet der Paderborner Hochfläche an die vom Cenoman-Pläner gebildeten Plateaus gebunden sind.

\subsubsection{Terra fusca-Relikte}

\subsubsection{Terra fusca-Relikte aus Kalksteinen des Cenoman 3}

Aus den hochprozentigen Kalksteinen des Cenoman $3\left(95 \% \mathrm{CaCO}_{3}\right.$ nach Arnold (1977, $91 \%$ a $\mathrm{CaCO}_{3}$ nach v. Zezschwitz 1964) sind im Gebiet der Paderborner Hochfläche während eines langen Verwitterungszeitraumes Böden vom Typ der Terra fusca hervorgegangen. Relikte dieser Böden sind nur örtlich auf erosionsfernen, isoliert gelegenen kleinen Plateaus erhalten geblieben. Ein solches von der Erosion wenigstens teilweise belassenes Vorkommen befindet sich auf dem Hayersberg, einem riedelartigen schmalen Plateau zwischen Altenau- und Piepenbachtal (vgl. Abb. 1). Mit einem mittleren Tonanteil von $63 \%$ stellt diese Terra fusca bodenartlich einen lehmigen Ton bis Ton dar (vgl. Tab. 1). Die hohe Sorptionskapazität von rund 29 mval/100 g Boden (vgl. Tab. 2) deutet auf erhebliche Anteile von Montmorin-Mineralien in der Tonfraktion hin. Sekun-

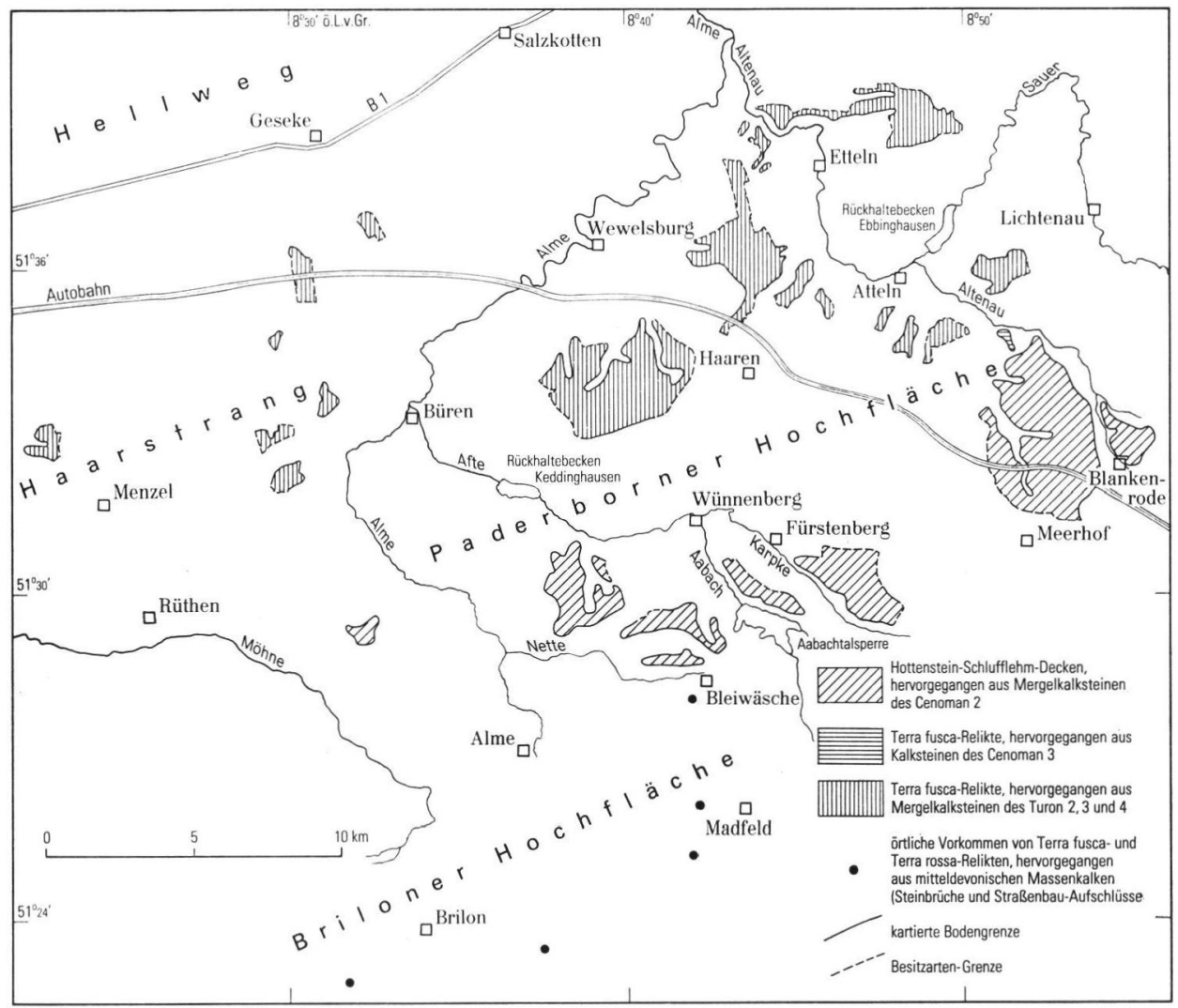

Abb. 1: Die Vorkommen von Paläoböden auf der Paderborner und der Briloner Hochfläche 
där eingemengtes carbonatisches Material (Kalkstein-Splitter) ist für die neutrale Bodenreaktion und die mit $89 \%$ festgestellte hohe Basensättigung des ursprünglich sicher saureren Terrae-Materials verantwortlich.

\subsubsection{Terra fusca-Relikte aus Mergelkalksteinen des Turon 2, 3 und 4}

Aus den Mergelkalksteinen des Turon 2, 3 und 4, die auf der Paderborner Hochfläche weiträumige Plateaus bilden, haben sich durchweg Böden vom Typ der Terra fusca entwickelt. Über ihre kartierte Verbreitung gibt Abb. 1 Auskunft. Gemessen am vergleichsweise geringen Lösungsrückstand der Kalksteine des Cenoman 3 ist bei der Lösungsverwitterung der turonen Mergelkalksteine, die einen Carbonatgehalt von rund $80 \%$ besitzen, bedeutend mehr bodenbildende Mineralsubstanz angefallen. Nach der Entstehung der saaleeiszeitlichen Frostschuttdecken dürften in den langen Verwitterungszeiträumen (vor allem im Eem) aus den Frostschuttresten deshalb ziemlich mächtige TerraeDecken entstanden sein, die vielleicht bis zu $2 \mathrm{~m}$ mächtig waren. Weichseleiszeitlich wurde deren Mächtigkeit solifluktiv jedenfalls auf 5 bis $10 \mathrm{dm}$ verkürzt. Die Terrae-Relikte wurden dann von den Lössen dieser Kaltzeit bedeckt. Ähnlich wie für die Deckschichten der Hottenstein-Schlufflehmdecken (unter 2.1.1. beschrieben), blieben von den äolischen Sedimenten nur schleierartig vorkommende Lehme als geringmächtige Decklehme auf den tonigen Reliktböden erhalten. Lediglich im Bereich von Mulden, Rinnen und an Unterhängen gehen diese Decklehme in Lößfließerden größerer Mächtigkeit über.

Hinsichtlich ihrer Körnung sind die aus turonen etwas tonärmer als die aus cenomanen Gesteinen entstandenen Terrae-Relikte (vgl. Tab. 1). Dies beruht wahrscheinlich auf weichseleiszeitlichen Umlagerungen, die sich auf den schwach geneigten großen TuronPlateaus besonders stark auswirken konnten. Wie nämlich mikromorphologische Befunde ${ }^{*}$ ) zeigen, besteht das Terrae-Material häufig aus einer innigen Vermengung von Braunlehm(Terra fusca-), grau gebleichtem ehemaligem Braunlehm- und aus Lößlehm-Material. Während die Braunlehm- und die (infolge von Staunässeeinflüssen) grau gebleichten Braunlehmanteile fast nur aus Ton bestehen, enthalten die Lößlehmanteile reichlich Quarzschluff sowie etwas Hornblende. Die verschiedenen Anteile sind in Form von Schlieren, Bröckchen (Tonkonglomerate) und in Geröllen (Ballengefüge) miteinander vermengt. Indizien also, die zusammen mit teilweise vorhandenen schwachen Schrägschichtungen das Terrae-Material als (solifluktiv) umgelagert ausweisen.

Wie die in Tab. 2 zusammengestellten Analysenwerte(Mittel von 37 Proben) zeigen, liegt die Sorptionskapazität der aus turonen Gesteinen hervorgegangene Terra fusca mit 26,5 mval nur wenig unter derjenigen der aus cenomanen Gesteinen hervorgegangenen Terrafusca. Dagegen sind S-Wert, Basensättigung und $\mathrm{pH}$-Wert sehr viel niedriger als bei dieser. Stellt man ferner den Carbonatgehalt der weichseleiszeitlich in die Terrae eingemengten Lösse in Rechnung, so dürften die eemzeitlichen Terrae-Bildungen ehemals sogar noch basenärmer gewesen sein. Röntgenographische Untersuchungen der Tonsubstanz ${ }^{* *}$ ) ergaben dann auch teilweise unerwartet hohe Gehalte an Koalinit (als Fireclay vorliegend), die bis zu 20\% betragen, ein Zeichen für intensive (basenzehrende) Verwitterungsprozesse in den Terrae-Decken.

Die weichseleiszeitliche Lößbedeckung der Terrae-Relikte muß ursprünglich nicht unerheblich gewesen sein. Auf den Turon-Plateaus konnten jedenfalls aus den Lößfließerden Parabraunerden entstehen, deren typisches $\mathrm{B}_{\mathrm{t}}$-Material im obersten Schüttgut bronzezeit-

*) Für die Durchführung der mikromorphologischen Untersuchungen wird Herrn Dr. F.-D. ERKWOH, Geologisches Landesamt Nordrhein-Westfalen, Krefeld, gedankt.

*) vgl. Fußnote Seite 205. 
licher Grabhügel nachgewiesen wurde (v. Zezschwitz 1980). Seit der mittleren Bronzezeit sind diese Bodenbildungen aus Lößlehm siedlungsbedingt bis auf nur mehr 2 bis $3 \mathrm{dm}$ mächtige Decklehme - die zudem stark der Pseudovergleyung unterlagen — abgetragen worden.

\subsection{Paläoböden der Briloner Hochfläche}

Für die im Bereich der Briloner Hochfläche gefundenen Paläoböden sind die Kreiderelikte auf dem Massenkalkplateau (Paeckelmann \& KüHne 1936 a, 1936b) von besonderem Interesse. Die cenomanen und präcenomanen Sedimente (Oberalb) reichen von Bleiwäsche im NE-Zipfel des Massenkalkplateaus (445 m über NN) mit Unterbrechungen inselartig bis nach Radlinghausen ( $470 \mathrm{~m}$ über NN). Nach dem Vorkommen und der Verbreitung der zahlreichen verkieselten Kreide-Blöcke zu urteilen - sie stellen Verwitterungsbildungen der jungkretazisch-tertiären Landoberfläche dar — haben die Kreidesedimente ursprünglich das gesamte Plateau bedeckt. Seit der Kreidezeit kann somit keine bedeutende flächenhafte Abtragung stattgefunden haben. Paeckelmann (1931 b) veranschlagt sie mit insgesamt nicht mehr als 30 Meter. Auch das von Clausen et al. (1978) festgestellte Fehlen von jungen oberkretazischen und älteren tertiären Karstsedimenten im Massenkalk der benachbart gelegenen Warsteiner Carbonatplattform spricht dafür, daß beide Massenkalkplateaus bis zum Jungtertiär noch von den Schichten der Kreide verhüllt waren. Mithin stellt die Briloner Hochfläche eine relativ junge pliozän-altpleistozäne Einebnungsfläche dar, die allerdings präcenoman schon weitgehend vorgeprägt war. Vorgefundene Paläoböden sind deshalb entweder plombierte und dann exhumierte präcenomane Bildungen oder sie sind erst im Jungtertiär oder Pleistozän entstanden.

Diese Paläoböden werden nachfolgend - im Westteil der Briloner Hochfläche beginnend - der Reihenfolge ihrer Fundpunkte nach (vgl. Abb. 1) beschrieben. Sie liegen am Süd- und Ostrand des Briloner Massenkalkplateaus.

In dem südwestlich von Brilon gelegenen, aufgelassenen Kalkspatbruch (B1. 4617 Brilon, R 346739, H 569400) findet sich bis in 29 m Tiefe auf Klüften und Schichtfugen des Eskesberger Kalkes ein leuchtend rot gefärbter Ton. Nach dem Ergebnis der Schlämmanalyse entfallen $79 \%$ des Feinbodens auf die Tonfraktion, ein auch für Tonböden ungewöhnlich hoher Tongehalt. Die mit 16,6 mval ermittelte Sorptionskapazität ist dagegen auffällig niedrig. Wenn auch Eisen- und Tonmineralbesimmungen sowie andere weiterführende Untersuchungen noch fehlen, sprechen doch bereits die Fundumstände angesichts des vorliegenden Kalkgesteins (der Briloner Massenkalk besitzt nach VogLeR 1977 mindestens $98 \% \mathrm{CaCO}_{3}$ ) dafür, daß es sich um Relikte einer präcenomanen Terra rossa handelt. Ahnliche, ebenfalls noch nicht identifizierte Bildungen sind übrigens von CLAusEN et al. (1978) im Paläokarst des Warsteiner Massenkalkes unter glaukonitführenden Sedimenten gefunden worden. Möglicherweise sind diese Paläoboden-Relikte auch mit den von MeINecke (1963) beschriebenen Terra rossa-Bildungen der weiter westlich gelegenen Massenkalkplateaus des Sauerlandes vergleichbar, die allerdings pliozänen Alters sein sollen.

Das zweite Terrae-Vorkommen befindet sich ostwärts von Brilon am Hangfuß des Romberges (Bl. 4617 Brilon, R 347407, H 569504). Der Fundpunkt liegt in unmittelbarer Nähe der südlichen Verbreitungsgrenze des Massenkalks, unweit des Hauptgrünsteinzuges, der von der Cenoman-Transgression nicht überschritten wurde (PAECKELMANN 1936). In diesem etwa $500 \mathrm{~m}$ über NN gelegenen Gebiet wurde der Massenkalk von der (nach $\mathrm{N}$ ) rückschreitenden Erosion im Jungtertiär zuerst von den cenomanen Ablagerungen befreit und lag wahrscheinlich bereits zu Beginn des Pliozäns als bodenbildendes Substrat für die Verwitterung frei. 
Die hier in Spalten und Taschen bis in Tiefen von $1 \mathrm{~m}$ gefundenen Terrae-Relikte bestehen aus lehmigem Ton $(56 \%<0,002 \mathrm{~mm})$, der rotbraun gefärbt ist und bei hoher Sorptionskapazität (31,6 mval) und Basensättigung $(78 \%$ ) ein kleinpolyedrisches Gefüge aufweist. Die mit 6 Gew.\% bestimmten Sandanteile des Feinbodens bestehen aus Quarz, der den Basisschichten der ehemaligen Kreideüberdeckung entstammen dürfte. Der hohe Steingehalt des Terrae-Materials (Massenkalk) läßt auf pleistozäne Vermengungen mit Frostschutt schließen. Der für reines Terrae-Material zu niedrige Tongehalt legt die Vermutung nahe, daß außerdem Löß eingemengt worden ist. Aufgrund der genannten Fundumstände wird das Paläoboden-Relikt als eine jungtertiär-altpleistozäne Bildung angesehen, ähnlich den Terrae-Relikten des Frankenwaldes, die Strebel (1961) ins Pliozän stellt.

In dem südsüdostwärts vom Stemmel gelegenen Steinbruch (B1. 4518 Madfeld, R 347980, H 569895) wurde in etwa $40 \mathrm{~m}$ Tiefe Terrae-Material geborgen, das den Massenkalk in Schlottenwandungen 1 bis $2 \mathrm{~cm}$ dick bekleidete. Die im Feinboden mit 20 Gew. $\%$ bestimmten „Sand“-Anteile erwiesen sich als Kalkstein-Splitter. Sie sind als Indiz für Umlagerung (Einwaschung in den Karst) zu werten. Legt man diese carbonatischen Feinstgrusanteile rechnerisch auf die Ton- und Schlufffraktionen der ermittelten Körnung um, ergibt sich, daß das Terrae-Material aus einem Ton besteht, dessen auf die Tonfraktion entfallender Anteil über 70\% liegt. Der blaßgelb gefärbte Ton besitzt mit 8,1 mval eine ganz ungewöhnlich niedrige Sorptionskapazität.

Infolge von Straßenbauarbeiten war die Oberfläche des Massenkalkes an der von Madfeld nach Radlinghausen führenden Straße im Jahre 1972 gut aufgeschlossen, vor allem südöstlich der Höhe 488,6 m. Straßenböschungen und Kabelgräben zeigten hier auf mehr als $300 \mathrm{~m}$ Länge unter einer im wesentlichen aus Lößlehm bestehenden Deckschicht zahlreiche bis zu $2 \mathrm{~m}$ in den Massenkalk reichende zapfenartige Karsttaschen und Spalten. Die Taschenfüllungen bestanden aus bräunlichgelben und leuchtend hellgelben Tonen, vermengt mit etwas Sand und Kies (Quarze und Lydite). Bei Tongehalten von $>70 \%$ und sehr hoher Sorptionskapazität (bis zu $33 \mathrm{mval}$ ) sind diese Bildungen als fossile Paläoboden-Relikte zu klassifizieren. Die fraglos aus den sandig-kiesigen Basisschichten der ehemaligen Kreidebedeckung stammenden, sekundär in das Terrae-Material eingemengten Sande und Kiese legen ein präcenomanes Alter dieser Paläoboden-Relikte nahe. Bedenkt man ferner, daß im nahen Radlinghausen die ungestört gelagerten basalen Kreideschichten in $470 \mathrm{~m}$ Höhe anstehen, also nur etwa $10 \mathrm{~m}$ tiefer als am Aufschluß, wird deutlich, daß die Reliktböden wahrscheinlich exhumierte, bis ins Pliozän plombierte präcenomane Bildungen darstellen.

In dem bei Bleiwäsche gelegenen Steinbruch der Firma Eley (Bl. 4518 Madfeld, R 347917, H 570359) wurden ebenfalls Terrae-Relikte gefunden. Dieses teilweise bis zu $20 \mathrm{~m}$ in die Karstschlotten eingespülte Material besitzt ähnliche Eigenschaften wie die Schlottenauskleidungen im Steinbruch am Stemmel. Hier wie dort erweisen sich die „Sand“-Anteile carbonatisches Feinbodenmaterial. Die Sorptionskapazität steht mit 14,6 mval zwar in keinem so schroffen Gegensatz zum stark tonigen Bodenmaterial wie am Stemmel, doch ist sie ebenfalls recht niedrig.

Fundumstände und Analysenwerte der bisher im Bereich der Briloner Hochfläche geborgenen Paläoböden lassen die Genese dieser Terrae-Relikte gleichsam schlaglichtartig erkennen. Im Gegensatz nämlich zu den auf der Paderborner Hochfläche großflächig an der heutigen Landoberfläche vorkommenden Terrae-Bildungen und Hottenstein-Decken, die im Pleistozän entstanden sind, dürften die im Massenkalk der Briloner Hochfläche verborgenen Paläosol-Relikte zwar teilweise tertiären, überwiegend aber präcenomanen Alters sein. 


\section{Schriftenverzeichnis}

Arnold, H. (1965): Einige gesteinsphysikalische Daten vom Hottenstein. - Decheniana 118: 107-108, 1 Tab.; Bonn.

- (1977): Erläuterungen zu Blatt C 4314 Gütersloh. - Geol. Kt. Nordrh.-Westf. 1:100 000: 156 S., 31 Abb., 10 Tab., 1 Taf.; Krefeld.

Clausen, C. D., Grebe, H., Leuteritz, K. \& Wirth, W. (1978): Zur Altersstellung und paläogeographischen Bedeutung des Paläokarstes auf der Warsteiner Carbonatplattform. - N. Jb. Geol. Paläont. Mh. H. 10: 577-589, 5 Abb.; Stuttgart.

FINKE, L. (1971): Die Verwertbarkeit der Bodenschätzungsergebnisse für die Landschaftsökologie, dargestellt am Beispiel der Briloner Hochfläche. - Bochumer Geograph. Arb., 10: 84 S., 10 Tab.; Paderborn.

Hesemann, J. (1975): Geologie Nordrhein-Westfalens. - 416 S., 255 Abb., 122 Tab., 11 Taf.; Paderborn (Schöningh).

Lotze, F. (1951): Das Quartär der Hellwegebene bei Geseke. - N. Jb. Geol. Paläont. Mh.; 33-38; Stuttgart.

Meinecke, F. (1963): Das Vorkommen von Terrarossa und Gelblehm auf Massenkalk im Sauerland. - Z. dt. geol. Ges., 115: 715-726, 4 Abb.; Hannover.

MNich, J. (1979): Das mittlere Diemeltal bei Marsberg und seine angrenzenden Hochflächen. Diss., Univ. Bonn: 355 S., 74 Abb.; Bonn.

Mückenhausen, E. \& Wortmann, H. (1958): Erläuterungen zur Bodenübersichtskarte von Nordrhein-Westfalen 1:300 000. - 144 S., 7 Tab., 10 Taf.; Krefeld (Geol. L. A. NordrheinWestf.).

PAAS, W. \& v. Zezschwitz, E. (1972): Erläuterungen zur Bodenkarte 1:10000 des Staatl. Forstamtes Wünnenberg. - 21 S., 2 Abb., Ber. Arch. Geol. L. A. Nordrh.-Westf., Krefeld. [Unveröff.].

Paeckelmann, W. (1931 a): Der Massenkalkboden von Brilon in Westfalen. - Mitt. Lab. Preuß. Geol. L. A. 13,2: 20 S., 6 Tab.; Berlin.

- (1931 b): Die Rumpffläche des Nordöstlichen Sauerlandes. - Jb. Preuß. Geol. L. A., 52: 472-519, 2 Taf., 1 Kt.; Berlin.

- (1936): Erläuterungen zur Geol. Kt. 1:25000 Bl. 4617 Brilon. - 67 S., 3 Abb.; Berlin (Preuß. Geol. L. A.).

— \& KüHNE, F. (1936 a): Erläuterungen zur Geol. Kt. 1:25000 Bl. 4517 Alme. - 61 S., 1 Abb., 1 Tab.; Berlin (Preuß. Geol. L. A.).

_ \& - (1936b): Erläuterungen zur Geol. Kt. $1: 25000$ Bl. 4518 Madfeld. - 79 S., 1 Tab.; Berlin (Preuß. Geol. L. A.).

Stille, H. (1903): Geologisch-hydrologische Verhältnisse im Ursprungsgebiete der Paderquellen zu Paderborn. - 129 S., 3 Abb., 6 Taf.; Berlin (Preuß. Geol. L. A.).

Strebel, O. (1961): Tertiäre Bodenbildungen und Verwitterungsreste im Frankenwald. - Geol. Jb., 78: 609-620, 1 Taf.; Hannover.

Scherp, A. (1979): Petrographische Untersuchung eines Hottensteins und seines Herkunftsgesteins, des Cenoman-Pläners. - 7 S., 2 Abb.; Ber. Arch. Geol. L. A. Nordrh.-Westf., Krefeld [Unveröff.].

VogLer, H. (1977): Nutzbare Festgesteine in Nordrhein-Westfalen. - 65 S., 11 Abb., 1 Tab., 1 Taf., Krefeld (Geol. L. A. Nordrh.-Westf.).

von Zezschwitz, E. (1958): Erläuterungen zur Bodenkarte 1:10 000 des Gemeindewaldes Nettelstädt. - 18 S., 5 Tab.; Arch. Geol. L. A. Nordrh.-Westf., Krefeld. — [Unveröff.].

- (1964): Erläuterungen zur Bodenkarte 1:10000 des Staatl. Forstamtes Dalheim. - 30 S., 4 Abb., 2 Tab.; Arch. Geol. L. A. Nordrh.-Westf., Krefeld. - [Unveröff.].

- (1967): Zur Geschichte und Vergesellschaftung typischer Böden der Paderborner Hochfläche. - Decheniana, 118: 222-234, $5 \mathrm{Abb}$.; Bonn.

- (1970): Erläuterungen zur Bodenkarte 1:10000 des Staatl. Forstamtes Böddeken. - 24 S., 1 Abb., 2 Tab.; Arch. Geol. L. A. Nordrh.-Westf., Krefeld. - [Unveröff.].

- (1975): Erläuterungen zur Bodenkarte 1:10 000 des Stiftsforstes Büren. - 20 S.; Arch. Geol. L. A., Nordrh.-Westf., Krefeld. - [Unveröff.].

- (1980): Reliktisches und jungholozänes Tonfließplasma in bronzezeitlichen Grabhügeln auf der Paderborner Hochfläche. - Fundber. aus Hessen, 20 (FISCHER-Festschr.): 423—447, 2 Abb., 4 Tab., 5 Taf.; Wiesbaden. 